\title{
Raman Shifts of Diamond Inclusions - A Possible Barometer
}

\author{
Izraeli, E. ${ }^{1}$, Wilcock, I.C. ${ }^{2}$, Navon, O. ${ }^{1}$
}

1. Institute of Earth Sciences, The Hebrew University, Jerusalem, 91904

2. Renishaw plc, Old Town, Wotton-under-Edge, Gloucestershire, GL12 7DH, UK.

Pressures and temperatures at the diamond source region are commonly estimated from the chemistry of mineral inclusions trapped in the diamonds during their formation, using appropriate thermodynamical equilibria for major and trace elements. Both pressure and temperature may be estimated for the peridotitic assemblage, but in the case of eclogitic diamonds, no suitable barometer was established. As the inclusions must be liberated from their host diamond prior to analysis, these methods are destructive.

Information about the conditions at the source region is also preserved in the internal pressure within diamond inclusions and in the deformation of the diamond lattice around them. As first noted by Sorby and Butler (1869) and Rosenfeld and Chase (1961), many inclusions are surrounded by birefringence halos, suggesting that the diamond is stressed by the inclusions. This deformation is the result of the different bulk modulus $(\mathrm{K})$ and thermal expansion coefficient $(\alpha)$ of the diamond and the trapped mineral inclusions. Harris et al. (1970) and Liu et al. (1990) used x-ray and Raman spectroscopy, respectively, in attempts to determine the internal pressure within garnet inclusions and to estimate the conditions during trapping of these inclusions in the growing diamond. We follow this line of research and report successful determinations of the internal pressure in and around olivine inclusions in diamonds.

Theory: Consider a small, spherical, isotropic inclusion trapped in a diamond at $\mathrm{P}_{0}$ and $\mathrm{T}_{0}$. We assume that no deformation was induced by the initial trapping, and the diamond did not deform plastically. Upon transition to the Earth's surface both the inclusion and the host diamond expand as pressure is released and contract with falling temperatures. If the final volume of the inclusion is smaller than that of its hole in the diamond (which behaves as the diamond itself), then the inclusion is under no pressure (as was the case for the garnets studied by Liu et al., 1990). If this is not the case, than the diamond is elastically deformed and the inclusion is under pressure. For spherical, isotropic inclusions that are small relative to the host diamond:

$$
\int_{\mathrm{P}_{0}}^{1 \text { bar }} \frac{1}{\mathrm{~K}_{\mathrm{d}}\left(\mathrm{P}, \mathrm{T}_{0}\right)} \mathrm{dP}+\int_{\mathrm{T}_{0}}^{300 \mathrm{~K}} \alpha_{\mathrm{d}}(1 \mathrm{bar}, \mathrm{T}) \mathrm{dT}-\int_{\mathrm{P}_{0}}^{\mathrm{P}_{1}} \frac{1}{\mathrm{~K}_{\mathrm{i}}\left(\mathrm{P}, \mathrm{T}_{0}\right)} \mathrm{dP}-\int_{\mathrm{T}_{0}}^{300 \mathrm{~K}} \alpha_{\mathrm{i}}\left(\mathrm{P}_{\mathrm{i}}, \mathrm{T}\right) \mathrm{dT}=\frac{3\left(\mathrm{P}_{\mathrm{i}}-1 \text { bar }\right)}{4 \mu_{\mathrm{d}}}
$$

where the subscripts $\mathrm{d}$ and $\mathrm{i}$ represent the diamond and the inclusion, respectively, $\mathrm{K}$ is the isothermal bulk modulus, $\alpha$ is the termal expansion coefficient, $\mu_{\mathrm{d}}$ is the shear modulus of diamond, and $\mathrm{P}_{\mathrm{i}}$ is the pressure exerted by the elasticaly deformed diamond on the inclusion. More elaborate solution may be obtained for non-spherical inclusions (Eshelby, 1957). If $K, \alpha$, and $P_{i}$ are known, then, eq. 1 defines a line of "zero volume difference" in a P-T space. This line defines the temperatures $\left(T_{0}\right)$ under which the hole in the diamond and the mineral in the inclusion are of exactly the same size under the same lithostatic pressure, $\mathrm{P}_{0}$.

Raman Spectroscopy: We used a Renishaw 1000 Raman microscope to measure the exact position of the $856 \mathrm{~cm}^{-1}$ olivine Raman line of three olivine inclusions in diamond and a number of olivine of different forsterite content. Fig. 1 shows that the line position of olivine vary with composition, and that the inclusions are shifted to higher wavenumbers relative to the free crystals (assuming that the trapped olivines fall in the normal range of $\mathrm{Fo}_{92}-\mathrm{Fo}_{94}$ ). The measured shifts are $0.6,1.5$ and 1.6 
$\mathrm{cm}^{-1}$. Using available measurements of the shift of the $856 \mathrm{~cm}^{-1}$ olivine Raman line with pressure (3.27 $\mathrm{cm}^{-1} / \mathrm{GPa}$, Chopelas, $1990 ; 3.09 \mathrm{~cm}^{-1} / \mathrm{GPa}$, Wang et al., 1993) we calculate $\mathrm{P}_{\mathrm{i}}$ of $0.19,0.49$, and $0.52 \mathrm{GPa}$, respectively for the three inclusions.

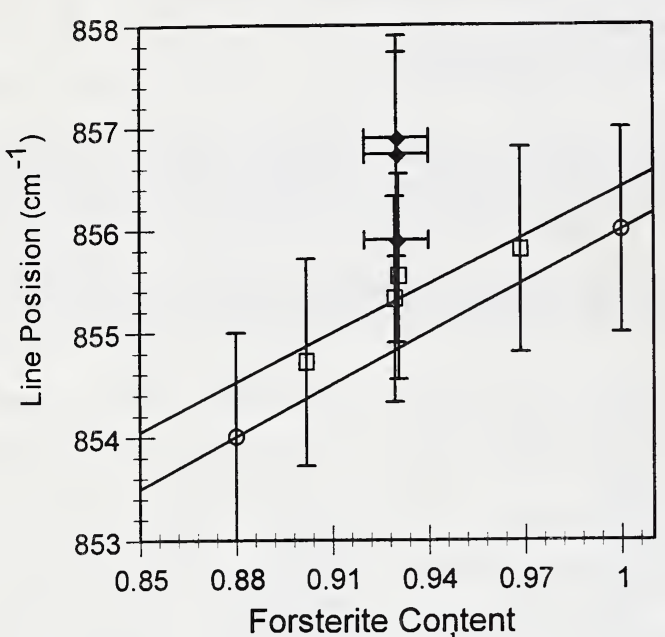

Fig. 1. The position of the $856 \mathrm{~cm}^{-1}$ line of olivine as a function of composition. Open squares - line position of four natural olivines measured by us. Circles - two olivines measured by Chopelas (1991). The slope is similar, the shift is due to calibration. Diamonds - line position of three olivine inclusions in diamonds (assuming composition of Fo=93 \pm 1 ). The three inclusions and the four free olivines were measured with the same spectrometer setting at the same day.

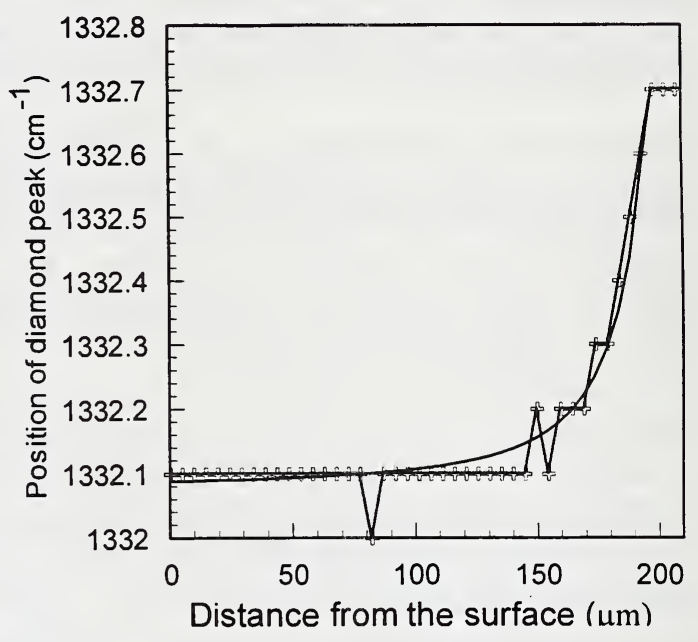

Fig. 2. The Raman line of diamond as a function of distance from the diamond surface (crosses). As the beam focuses closer to an olivine inclusion the peak position shifts to higher values due to the stress by the inclusion. The shift decreases with increasing distance according to $1 / \mathrm{r}^{2}$ (line).

More information about the deformation around the inclusion is in the diamond, as is evident from the birefringence around inclusions. The Raman line of the diamond allows quantitative study of this deformation. The diamond is under radial compression and tangential tension. Under uniaxial stress the diamond line shifts to higher frequencies (Grimsditch et al., 1978). A preliminary measurement of the shift of the diamond line as a function of distance from one of the inclusions is presented in Fig. 2. The profile follows the predicted $1 / \mathrm{r}^{2}$ dependence predicted for the stress in the diamond (Kirsch, 1898), the maximum shift of $0.6 \mathrm{~cm}^{-1}$ corresponds to applied stress of $\sim 0.27 \mathrm{GPa}$ (using $2.2 \mathrm{~cm}^{-1} / \mathrm{GPa}$ Grimsditch et al., 1978), within the pressure range indicated by the shifts in the olivine line.

Determination of pressure: Using Eq. 1, with literature values of $\mathrm{K}$ and $\alpha$, we calculate lines of "zero volume difference" for different values $\mathrm{P}_{\mathrm{i}}$ (Fig. 3). For a typical temperature of $1000^{\circ} \mathrm{C}$, and internal pressures of $0.2-0.5 \mathrm{GPa}$, the calculated pressure of origin is $5.4-5.8 \mathrm{GPa}$, in agreement with thermodynamical estimation of the conditions for the source region of ultramafic diamonds. Fig. 3 also demonstrate the high precision of the method. The almost linear dependence may be approximated by $\mathrm{P}_{0}(\mathrm{GPa})=-1.3+0.005 \mathrm{~T}(\mathrm{~K})+1.4 \mathrm{P}$, so the precision of the calculated pressure is similar to that of the measured pressure. Precision of $\pm 0.5 \mathrm{~cm}^{-1}$ in the shift corresponds to $\pm 0.2 \mathrm{GPa}$ ( $2 \mathrm{kbar}$ ) in pressure at the origin. The accuracy of the method depends mainly on that of $\mathrm{K}$ and $\alpha$, and on the strain history of the diamond (which is assumed to be fully elastic). 
We conducted similar calculations for other common phases in diamonds. Garnet inclusions are expected to be under no pressure (in accordance with the results of Liu et al. 1990), unless they were trapped under pressures greater than $6.5 \mathrm{GPa}\left(\right.$ at $\left.1000^{\circ} \mathrm{C}\right)$. In the case of pyroxenes, the dependence of $\mathrm{K}$ on temperature is not known. Assuming variation as in olivine, pyroxenes trapped at pressures exceeding 4-4.5 $\mathrm{GPa}\left(\right.$ at $1000^{\circ} \mathrm{C}$ ) are expected to be under pressure. Unfortunately, the slopes of the "zero volume difference" lines of different minerals are similar. This does not allow precise determination of $\mathrm{P}$ and $\mathrm{T}$ from intersecting lines. The shallow slopes indicate that the lines of "zero volume difference" are good barometers and intersections with thermodynamical thermometers would yield precise $\mathrm{P}$ and $\mathrm{T}$ of the source region.

Raman spectroscopy of eclogitic garnet or clinopyroxene combined with the Ellis and Green (1979) thermometer may allow determination of the depth of origin of eclogitic diamonds. The method may also be used for obtaining independent pressure estimates for "ultra-deep" diamonds.

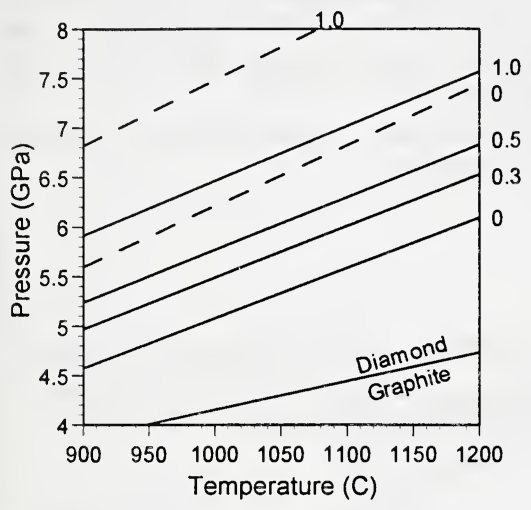

Fig. 3. Calculated pressures at the origin, as a function of temperature along lines of "zero volume difference" for olivine (solid lines) and garnet (dotted lines). Numbers to the right of the lines denote the present internal pressure in the inclusion, $\mathrm{P}_{\mathrm{i}}$. Inclusions trapped at pressures and temperatures below the $\mathrm{P}_{\mathrm{i}}=0$ lines will be under no internal pressure.

\section{References:}

Chopelas, A., 1990, Thermal properties of forsterite at mantle pressures derived from vibrational spectroscopy. Phys. Chem. Minerals, 17, p. 149-156.

Chopelas, A., 1991, Single crystal Raman spectra of forsterite, fayalite and monticellite. Amer. Mineral., 76, p. 1101-1109.

Ellis, D.J. and Green, D.H., 1979, An experimental study of the effect of Ca upon garnet-clinopyroxene Fe-Mg exchange equilibria. Contrib. Mineral. Petrol., 71, p. 13-22.

Eshelby, J.D., 1957, The determination of the elastic field of an ellipsoidal inclusion, and related problems. Proc. Royal. Soc., A 241, p. 376-396.

Grimsditch, M.H., Anastassakis, E. and Cardona, M., 1978, Effect of uniaxial stress on the zone-center optical phonon of diamond. Phys. Rev. B, 18, p. 901-904.

Harris, J.W., Milledge, H.J., Barron T.H.K. and Munn, R.W., 1970, Thermal expansion of garnets included in diamond. J. Geophys. Research, 75, p. 5775-5791.

Kirsch, D., 1898, Die theorie der elastizitat und die beaurforisse der festigkeitslehre, V DI Z , 42, p. 707-731.

Liu, L.G., Mernagh, T.P. and Jaques A.L., 1990, A mineralogical Raman spectroscopy study on eclogitic garnet inclusions in diamonds from Argyle. Contrib. Mineral. Petrol., 105, p. 156-161.

Rosenfeld, J.L. and Chase, A.B., 1961, Pressure and temperature of crystallization from elastic effects around solid inclusions in minerals? Amer. J. Sci., 259, p. 519-541.

Sorby, H.C. and Butler, P.J., 1869, On the structure of rubies, sapphire, diamonds and some other minerals. Royal. Soc. Lond. Proc., 17, p. 291-302.

Wang, S.Y., Sharma, S.K. and Cooney T.F., 1993, Micro-Raman and infrared spectral study of forsterite under high pressure. Amer. Mineral., 78, p. 469-476. 